\title{
Utility of Photoplethysmography for Heart Rate Estimation among Inpatients
}

Anoop N Koshy, MBBS, ${ }^{a}$ Jithin K Sajeev, MBBS, ${ }^{a}$ Nitesh Nerlekar, MBBS, MPH, ${ }^{b}$ Adam J Brown, MBBS, PhD, ${ }^{\mathrm{b}}$ Kevin Rajakariar, MBBS, ${ }^{a}$ Mark Zureik, MBBS, ${ }^{a}$ Michael C Wong, MBBS, PhD, ${ }^{a}$ Louise Roberts, PhD, ${ }^{a}$ Maryann Street, $\mathrm{PhD},{ }^{\mathrm{a}, \mathrm{c}}$ Jennifer Cooke, MBBS, ${ }^{a}$ Andrew W Teh MBBS, PhD ${ }^{\mathrm{a}, \mathrm{d}}$

\section{Affiliations:}

aMonash University, Eastern Health Clinical School, Department of Cardiology, Box Hill, Victoria, Australia

${ }^{b}$ Monash Cardiovascular Research Centre, Department of Medicine, Monash University and Monash Heart, Monash Health, Clayton, Victoria, Australia

'Deakin University, Geelong, Victoria, Australia

${ }^{\mathrm{d}}$ The University of Melbourne, Austin Hospital Clinical School, Department of Cardiology, Heidelberg, Victoria, Australia

Funding: This work was supported by the Eastern Health Research Foundation Grant. Dr. Nerlekar is supported by the National Health and Medical Research Council of Australia and National Heart Foundation Scholarship. Dr. Teh is supported by an Early Career Fellowship from the National Health and Medical Research Council of Australia.

Disclosures: All authors have reported that they have no relationships relevant to the contents of this paper to disclose.

\section{Word Count: 1411}

\section{Address for Correspondence:}

Dr. Andrew W Teh MBBS, PhD

Monash University, Eastern Health Clinical School

Department of Cardiology, Box Hill Hospital

5 Arnold Street, Box Hill 3128

Victoria, Australia

T: +614 22892015

F: +61398954852

E: Andrew.teh@easternhealth.org.au

\section{Trial Registration: Australia New Zealand Clinical Trials Registry} 12616001374459

Key Words: photoplethysmography, Arrhythmias, Atrial Fibrillation, Heart Rate, Accuracy

This is the author manuscript accepted for publication and has undergone full peer review but has not been through the copyediting, typesetting, pagination and proofreading process, which may lead to differences between this version and the Version of Record. Please cite this article as doi: $10.1111 / \mathrm{imj} .13777$

This article is protected by copyright. All rights reserved. 


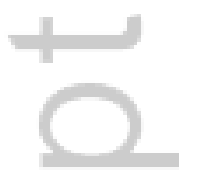




\begin{abstract}
The accuracy of photoplethysmography (PPG) for heart rate (HR) estimation in cardiac arrhythmia is unknown. PPG-HR was evaluated in 112 hospitalized inpatients (cardiac arrhythmias $(n=60)$, sinus rhythm $(n=52))$ using a continuous electrocardiogram monitoring as a reference standard. Strong agreement was observed in sinus rhythm $\mathrm{HR}<100$ and atrial flutter (bias 1 beat), modest agreement in sinus tachycardia (bias 24 beats) and complete heart block (bias -6 beats) and weak agreement with significant HR underestimation was seen in atrial fibrillation (bias 23 beats). Routine utilisation of PPG for HR estimation may delay early recognition of clinical deterioration in certain arrhythmias and sinus tachycardia.
\end{abstract}




\section{Introduction}

Heart rate (HR) measurement by photoplethysmography (PPG) technology has clinical appeal as a rapid, low cost alternative to a manual pulse-check or a standard electrocardiogram (ECG) in ward based settings. ${ }^{1,2}$ PPG uses an infrared lightemitting diode and photodetector to monitor blood volume changes in the microvasculature where each pulsatile signal is interpreted as an $\mathrm{R}$ wave. ${ }^{1}$ Although current nursing guidelines recommend 60-second manual pulse-counts during vital sign assessment, less than $25 \%$ of nurses adhere to this recommendation. ${ }^{3} \mathrm{HR}$ estimation using PPG has been validated among patients in sinus rhythm but a paucity of data exists to support its use in cardiac arrhythmias. ${ }^{1,4}$ With an increasing prevalence of atrial fibrillation (AF) among hospitalized patients, attaining recommended HR targets with titration of chronotropic therapies has prognostic implications especially among patients with heart failure. ${ }^{5,6}$ Furthermore, early detection of significant brady or tachyarrhythmia among inpatients not on continuous telemetry monitoring may facilitate timely intervention. ${ }^{7}$ The objective of this study was to compare the accuracy of HR assessment of common arrhythmias with a finger-based PPG monitor to continuous ECG monitoring as a reference standard.

\section{Methods}

Patients aged 18 years and over were prospectively recruited from the coronary care unit, intensive care unit and the emergency room. Two cohorts of patients were recruited based on underlying rhythm identified on 12-lead ECG: a) Arrhythmias b) Sinus Rhythm (SR). The arrhythmia cohort included patients with atrial arrhythmias and atrioventricular conduction disorders. Bradyarrhythmias and tachyarrhythmias were defined as mean heart rates $<60 \mathrm{bpm}$ and $\geq 100 \mathrm{bpm}$ respectively. Exclusion 
criteria included implantable cardiac devices, patients in contact isolation and a mechanical inability to secure the PPG monitor. The study was approved by the institutional review board and patients provided written informed consent (ACTRN: 12616001374459).

Standard five-lead ECG telemetry system (IntelliVue, Phillips, The Netherlands) was utilized as the reference standard on all subjects. The electrode placement sites were prepared by standardized procedures of cleaning, shaving and skin abrading to minimize artifact and five self-adhesive electrodes were placed on right arm, left arm, right leg, left leg and a V1 unipolar precordial lead. A hospital vital sign monitor (MP5 Monitor, Phillips Medical Systems, The Netherlands) with a photoplethysmograph was attached to the index finger. To exclude motion artefact, participants remained supine in bed for 30-minutes with HR data collected every 15-seconds (HR/15s) with a period of 1-minute at the start of the protocol disregarded, allowing for calibration of the devices.

Continuous variables are reported as mean \pm standard deviation. Bland-Altman plots with $95 \%$ upper and lower limits of agreement (LoA) were used to assess agreement between PPG-HR and ECG-HR. A narrower LoA range indicates higher agreement between the two variables. Bias indicates the mean difference between ECG-HR and SW-HR (HR/15s). Correlation was assessed using Spearman rank correlation $\left(r_{s}\right)$ due to non-Gaussian distribution. Two-sided $p$-values with a significance level of $<0.05$ was adopted. Statistical analyses were performed using Stata 14/MP (Statacorp, College Station, Texas, USA). 


\section{Results}

There were 112 consecutive patients recruited (65\% male; mean age $68 \pm 15$ years).

Sixty patients that were included in the arrhythmia cohort analysis include: atrial fibrillation (AF, $n=32)$, atrial flutter $(n=20)$ and complete heart block $(C H B, n=8)$. All CHB patients in the study were haemodynamically stable and monitored in coronary care prior to implantation of a permanent pacemaker. Fifty-two patients in SR, included those with persistent sinus bradycardia $(H R<60, n=20)$, sinus rhythm with HR 60-99 $(n=20)$ and persistent sinus tachycardia $(H R \geq 100, n=12)$.

Across both devices, $26,880 \mathrm{HR}$ values were recorded. Overall, the SR cohort with $H R$ within physiological range $(H R<100)$ demonstrated strong agreement with $E C G-$ HR with a mean bias of 1 beat and $r_{s} 0.96(p<0.001)$ (Figure 1). Despite the low overall mean bias observed in this cohort, wide LoA (-22 to 25 beats) observed suggests a degree of imprecision that may have been influenced by outlier results as seen on the the Bland-Altman plot. HR assessment in sinus tachycardia (HR $\geq 100 \mathrm{bpm}$ ), demonstrated a mean bias of 24 beats with wider LoA (-38 to 66 beats), suggesting marked HR underestimation with PPG.

Presence of any arrhythmia led to reduced agreement when compared with sinus rhythm (Table). Subgroup arrhythmia analysis demonstrated the highest agreement for atrial flutter (bias 1 beat, $95 \%$ LoA -8 to $10,\left(r_{s} 0.99, p<0.001\right)$ ). AF in particular, demonstrated poor agreement with a mean bias of 22 beats with wide LoA -34 to 80 beats suggesting HR underestimation and weak correlation $\left(r_{s} 0.31, p<0.001\right)$

(Figure 2). CHB subjects demonstrated a mean bias of -6 beats, with poor agreement as demonstrated by the wide LoA of -49 to 38 beats. 


\section{Discussion}

Our findings demonstrate two key findings: 1) Sinus rhythm with $\mathrm{HR}<100$ and atrial flutter demonstrated minimal bias and strong correlation with ECG HR; 2) CHB and sinus tachycardia demonstrated only moderate agreement with ECG derived HR with wider LoA; 3) Poor agreement, with significant HR underestimation was noted in AF.

Despite widespread adoption of PPG-based HR monitoring in hospitals, this is the first study evaluating their accuracy in arrhythmias. The difference in accuracy between AF and atrial flutter is not unexpected. Beat-to-beat variability in AF and reduced perfusion of the microvasculature can impair signal recognition and HR computation similar to the clinical concept of a 'pulse deficit'. ${ }^{8,9}$ In contrast, atrial flutter being a reentrant tachycardia would have minimal variability in HR and stroke volume, thereby attenuating HR underestimation due to device filtering algorithms. ${ }^{10}$ Findings of a wide LoA in CHB patients suggests a degree of variability with the PPG HR estimation in this cohort. The generalizability of this finding however, is limited by the small sample size.

The reduced HR agreement in sinus tachycardia is a novel finding, as patients in this study were evaluated at rest. HR underestimation during sinus tachycardia has been reported previously in laboratory-based exercise settings where motion artefact and impaired skin contact during peak exercise were thought to primarily affect HR computation. ${ }^{11,12}$ Our results taken in resting subjects suggests that tachycardia in itself may contribute to inherent inaccuracies in PPG based HR estimation due to effects on sampling frequency or signal filtering of the device. Alternatively, sinus 
tachycardia which often coexists in the context of sepsis, inotrope use or metabolic derangement could increase systemic vascular resistance and impair peripheral perfusion, thereby affecting HR computation of the photoplethysmograph.

Our results demonstrate that routine use of PPG in a hospital setting for estimating HR may produce inconsistent results. HR is currently incorporated in the Medical Emergency Team (MET) activation platform, which has reduced the incidence and mortality from in-hospital cardiac arrests. ${ }^{7}$ Given that tachycardia and arrhythmias including AF account for over $20 \%$ of MET activations, utilization of PPG alone might delay early identification of the deteriorating patient with tachycardia or AF. ${ }^{13}$ Furthermore, clinician decisions on titration of chronotropic agents are made based on HR trends documented in a patient's vital sign measurements. Inappropriate or inadequate alterations in pharmacotherapy based on PPG-derived HR readings could therefore also predispose to sub-optimal HR control or result in premature discharge of a patient with an underlying tachyarrhythmia.

Previous studies have reported the auscultatory method for 60 -seconds to be superior to a radial pulse check in AF or when HR exceeds 100 beats per minute..$^{3,14}$ A comparison of a manual pulse-check to PPG derived HR however, is lacking. New machine learning algorithms that utilize granular PPG data have shown promise in differentiating sinus rhythm from arrhythmias. ${ }^{11}$ Although incorporating these into conventional PPG monitors could improve their accuracy, this is yet to be proven. The widespread availability of smart watches and fitness trackers are an attractive prospect for estimation of HR using consumer-derived biometric data. However, 
given the poor accuracy of PPG which uses similar technology, patients should be cautioned on utilizing these until further validation studies are conducted.

\section{Limitations}

Only one brand of a medical-grade photoplethysmograph was assessed in this study and this may limit the generalizability of our findings. Secondly, the HR values were assessed when patients were immobile in bed and may not be representative of ambulant patients, where exercise and movement may exaggerate contact artifact. $^{2,12}$. This methodology however, was deliberately chosen to assess HR accuracy without confounding from movement artifact.

\section{Conclusion}

PPG has excellent agreement to ECG-HR in sinus rhythm $(H R<100)$ and atrial flutter, but significantly underestimates HR in AF and sinus tachycardia. Accurate assessment of HR is critical among any cohort of hospitalized patients, irrespective of their cardiac rhythm. As such, PPG should not replace a manual pulse assessment or ECG in assessing HR in patients that are not on continuous cardiac monitoring. 


\section{References}

1. Allen J. Photoplethysmography and its application in clinical physiological measurement. Physiol Meas. 2007;28(3):R1-39.

2. Sinex JE. Pulse oximetry: principles and limitations. Am J Emerg Med. 1999;17(1):59-67.

3. Hollerbach AD, Sneed NV. Accuracy of radial pulse assessment by length of counting interval. Heart Lung. 1990;19(3):258-64.

4. Carrara M, Carozzi L, Moss TJ, de Pasquale M, Cerutti S, Ferrario M, et al. Heart rate dynamics distinguish among atrial fibrillation, normal sinus rhythm and sinus rhythm with frequent ectopy. Physiol Meas. 2015;36(9):1873-88.

5. Zoni-Berisso M, Lercari F, Carazza T, Domenicucci S. Epidemiology of atrial fibrillation: European perspective. Clin Epidemiol. 2014;6:213-20.

6. Nikolovska Vukadinovic A, Vukadinovic D, Borer J, Cowie M, Komajda M, Lainscak $\mathrm{M}$, et al. Heart rate and its reduction in chronic heart failure and beyond. Eur J Heart Fail. 2017.

7. Buist MD, Moore GE, Bernard SA, Waxman BP, Anderson JN, Nguyen TV. Effects of a medical emergency team on reduction of incidence of and mortality from unexpected cardiac arrests in hospital: preliminary study. BMJ. 2002;324(7334):387-90.

8. Halmos PB, Patterson GC. Effect of atrial fibrillation on cardiac output. Br Heart J. 1965;27(5):719-23.

9. Carrara M, Carozzi L, Moss TJ, de Pasquale M, Cerutti S, Ferrario M, et al. Heart rate dynamics distinguish among atrial fibrillation, normal sinus rhythm and sinus rhythm with frequent ectopy. Physiol Meas. 2015;36(9):1873-88. 
10. Samet P. Hemodynamic sequelae of cardiac arrhythmias. Circulation. 1973;47(2):399-407.

11. Zhang Z, Pi Z, Liu B. TROIKA: a general framework for heart rate monitoring using wrist-type photoplethysmographic signals during intensive physical exercise. IEEE Trans Biomed Eng. 2015;62(2):522-31.

12. Iyriboz Y, Powers S, Morrow J, Ayers D, Landry G. Accuracy of pulse oximeters in estimating heart rate at rest and during exercise. $\mathrm{Br} \mathrm{J}$ Sports Med. 1991;25(3):162-64.

13. Jones D, Duke G, Green J, Briedis J, Bellomo R, Casamento A, et al. Medical emergency team syndromes and an approach to their management. Crit Care. 2006;10(1):R30.

14. Sneed NV, Hollerbach AD. Accuracy of heart rate assessment in atrial fibrillation. Heart Lung. 1992;21(5):427-33.

\section{Figure Legend}


Figure 1: Bland-Altman plot comparing PPG-HR to ECG monitoring (ECG-HR) in Sinus Rhythm. Black dotted line represents the mean difference (bias) between the two measures and red dashed lines are the $95 \%$ limits of agreement. Measures are HR recorded every 15 seconds. Plots demonstrate a bias of 1 beat with wide LoA (16 to 19$)$ suggesting a degree of HR variability.

\section{Figure 2:}

Top: Spearman rho correlations $\left(r_{s}\right)$ between Photoplethysmography Heart Rate (PPG-HR) and 5-Lead Electrocardiogram HR (ECG Monitoring) and for AF and Atrial Flutter. Measures are HR values recorded every 15 seconds.

Bottom: Bland-Altman plots comparing PPG-HR to ECG monitoring (ECG-HR) in AF and Atrial Flutter. Black dotted line represents the mean difference (bias) between the two measures and red dashed lines are the $95 \%$ limits of agreement. Measures are HR recorded every 15 seconds. Plots demonstrate smaller bias in atrial flutter (1 beat) compared to AF (23 beats) with small LoA (-8 to 10 vs -35 to 80 ).

Table 1: Accuracy of Photoplethysmography Estimated Heart Rate 


\begin{tabular}{llll}
\hline Rhythm & Correlation & Bias & $95 \%$ LOA \\
\hline $\begin{array}{l}\text { Atrial Fibrillation } \\
\text { ( } n=7,680)\end{array}$ & $0.31^{*}$ & 23 & -35 to 80 \\
\hline $\begin{array}{l}\text { Atrial Flutter } \\
\text { ( } n=4,800)\end{array}$ & $0.99^{*}$ & 1 & -8 to 10 \\
\hline $\begin{array}{l}\text { Complete Heart Block } \\
\text { (n=1,920) }\end{array}$ & $0.69^{*}$ & -6 & -50 to 38 \\
\hline $\begin{array}{l}\text { All Arrhythmias } \\
\text { (n=14,400) }\end{array}$ & $0.71^{*}$ & 12 & -39 to 62 \\
\hline $\begin{array}{l}\text { All Sinus Rhythm } \\
\text { (n=12,480) }\end{array}$ & $0.96^{*}$ & 1 & -16 to 19 \\
\hline $\begin{array}{l}\text { Sinus Rhythm HR } \\
<100 \\
\text { (n=9,600) }\end{array}$ & $0.98^{*}$ & 3 & -22 to 25 \\
\hline $\begin{array}{l}\text { Sinus Rhythm HR } \\
\geq 100 \\
\text { (n=2,880) }\end{array}$ & $0.69^{*}$ & 24 & -38 to 66 \\
\hline
\end{tabular}

*indicates $p<0.01$. Correlations are Spearman rho $\left(r_{s}\right)$ correlation co-efficient. Bias indicates heart rate difference between ECG-monitoring and Photoplethysmography, heart rates per values recorded every 15 seconds, with associated $95 \%$ limits of agreement (LOA) 


\section{University Library}

\section{- M M N E R VA A gateway to Melbourne's research publications}

Minerva Access is the Institutional Repository of The University of Melbourne

\section{Author/s:}

Koshy, AN;Sajeev, JK;Nerlekar, N;Brown, AJ;Rajakariar, K;Zureik, M;Wong, MC;Roberts,

L;Street, M;Cooker, J;Teh, AW

Title:

Utility of photoplethysmography for heart rate estimation among inpatients

\section{Date:}

2018-05-01

\section{Citation:}

Koshy, A. N., Sajeev, J. K., Nerlekar, N., Brown, A. J., Rajakariar, K., Zureik, M., Wong, M. C., Roberts, L., Street, M., Cooker, J. \& Teh, A. W. (2018). Utility of photoplethysmography for heart rate estimation among inpatients. INTERNAL MEDICINE JOURNAL, 48 (5), pp.587-+. https://doi.org/10.1111/imj.13777.

Persistent Link:

http://hdl.handle.net/11343/283969 